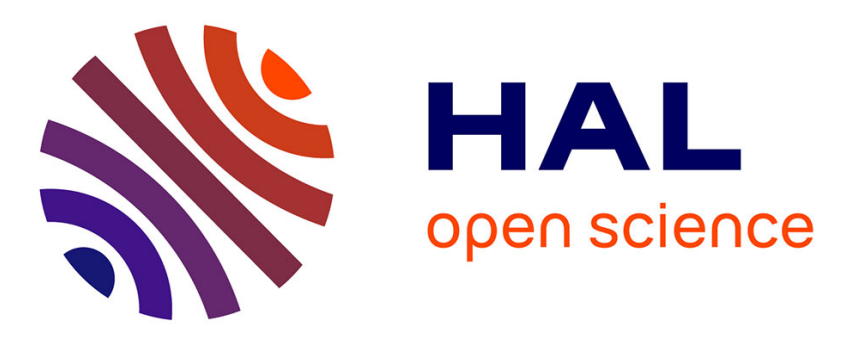

\title{
Double chambre d'ionisation alpha avec passeur d'échantillons
}

\author{
R. Bimbot, C. Deprun, D. Gardès, Y. Le Beyec
}

\section{To cite this version:}

R. Bimbot, C. Deprun, D. Gardès, Y. Le Beyec. Double chambre d'ionisation alpha avec passeur d'échantillons. Revue de Physique Appliquée, 1973, 8 (1), pp.97-98. 10.1051/rphysap:019730080109700 . jpa-00243660

\section{HAL Id: jpa-00243660 https://hal.science/jpa-00243660}

Submitted on 1 Jan 1973

HAL is a multi-disciplinary open access archive for the deposit and dissemination of scientific research documents, whether they are published or not. The documents may come from teaching and research institutions in France or abroad, or from public or private research centers.
L'archive ouverte pluridisciplinaire HAL, est destinée au dépôt et à la diffusion de documents scientifiques de niveau recherche, publiés ou non, émanant des établissements d'enseignement et de recherche français ou étrangers, des laboratoires publics ou privés. 
Classification

Physics abstracts

11.10

\title{
DOUBLE CHAMBRE D'IONISATION ALPHA AVEC PASSEUR D'ÉCHANTILLONS
}

\author{
R. BIMBOT, C. DEPRUN, D. GARDÈS et Y. LE BEYEC \\ Laboratoire de Chimie Nucléaire, Institut de Physique Nucléaire \\ B. P. no 1, 91-Orsay, France
}

(Reçu le 11 juillet 1972)

\begin{abstract}
Résumé. - Cet ensemble permet de mesurer successivement l'activité alpha de huit sources, deux d'entre elles étant comptées simultanément.

Abstract. - This device is designed to measure successively the alpha activity of eight samples, two of them being counted simultaneously.
\end{abstract}

Ce dispositif a été réalisé dans le but de doter une chambre d'ionisation à boule [1], [2] d'un passeur d'échantillons permettant de présenter successivement huit sources sans être obligé d'évacuer le gaz de remplissage de la chambre, ni de couper la haute tension appliquée entre la boule et le porte-source. Sans augmenter beaucoup le prix de revient de l'appareil, ni de l'électronique associée, il a été possible d'incorporer deux chambres d'ionisation dans la même enceinte. Un système à 4 chambres serait également concevable. Ces chambres d'ionisation ont pour but de mesurer la radioactivité de sources émettrices alpha de faible intensité avec une bonne efficacité $(0,5)$ et une résolution de l'ordre de $100 \mathrm{keV}$.

1. Description (cf. Fig. 1). - Les plans de constructions de cet appareil peuvent être obtenus sur demande au Bureau d'Etudes de l'Institut de Physique Nucléaire d'Orsay, où ils sont répertoriés sous le $\mathrm{n}^{\circ} 170100$.

L'enceinte ellipsoïdale, est en fer et son intérieur est microbillé. Le couvercle C s'ouvre en pivotant autour

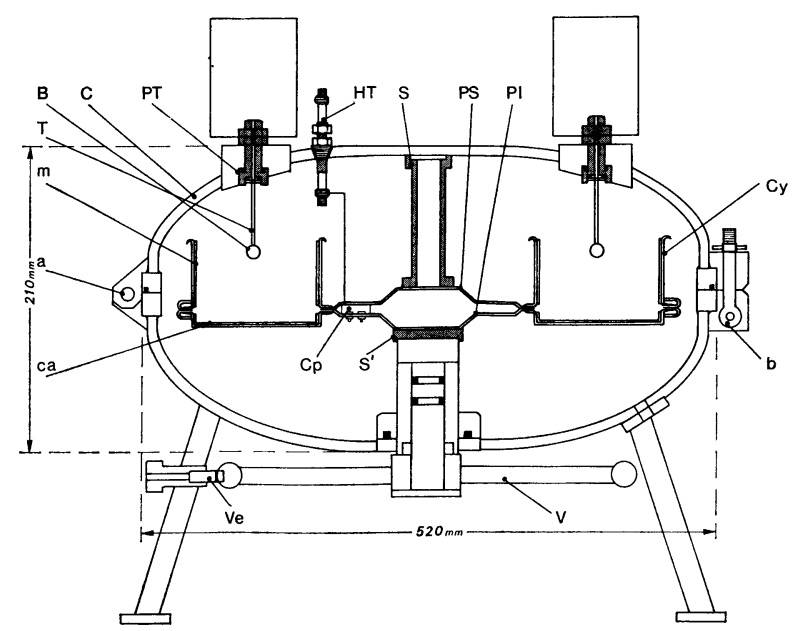

Fig. 1. - Schéma de la double chambre d'ionisation (voir texte). d'un axe a. En position fermée, le verrouillage est assuré par 12 boulons à œil $\mathrm{b}$ de diamètre $12 \mathrm{~mm}$ munis d'écrous papillons.

Le plateau supérieur PS, en cuivre, porte les parois cylindriques CY des deux chambres d'ionisation, et est fixé au couvercle par un support isolant $\mathrm{S}$ en afcodur. Le plateau inférieur PI, en cuivre également, et dans lequel sont creusées les 8 cuvettes est manœuvré de l'extérieur par un volant $\mathrm{V}$ isolé du plateau par la plaquette d'afcodur $S^{\prime}$. Le détail du passage tournant étanche est montré sur la figure 1 . Le volant est muni d'encoches permettant de le bloquer dans les 8 positions de comptage à l'aide du verrou Ve. La haute tension est amenée sur le plateau supérieur par un passage isolant HT étanche au vide et à la pression. Elle est transmise au plateau inférieur par des contacts en peigne de bronze au béryllium (CP).

Les impulsions sont recueillies sur les boules B, montées sur une tige de cuivre $\mathrm{T}$ qui assure la liaison électrique avec le préamplificateur, au moyen d'un passage isolé en téflon (PT). En cas de contamination, la boule peut être changée sans démonter le passage isolant. L'intérieur des chambres d'ionisation est doublé d'aluminium (manchon cylindrique $\mathrm{m}$ pour les parois, cuvette amovible (ca) pour le fond) que l'on peut changer également en cas de pollution.

Enfin, il existe une arrivée de gaz qui sert également de prise de vide, et une soupape de sécurité fixée sous la chambre.

Chacune des boules est reliée à une chaîne d'amplification (préamplificateur de charges, amplificateur d'impulsions) et à un analyseur multicanaux.

2. Conditions de fonctionnement et caractéristiques. - Le gaz de remplissage est l'argon contenant $3 \%$ de $\mathrm{CO}_{2}$. La haute tension appliquée sur les plateaux est égale à $5000 \mathrm{~V}$. La pression donnant l'optimum de résolution varie de 2 à 3 atmosphères suivant l'énergie des raies alpha mesurées. La résolution opti- 


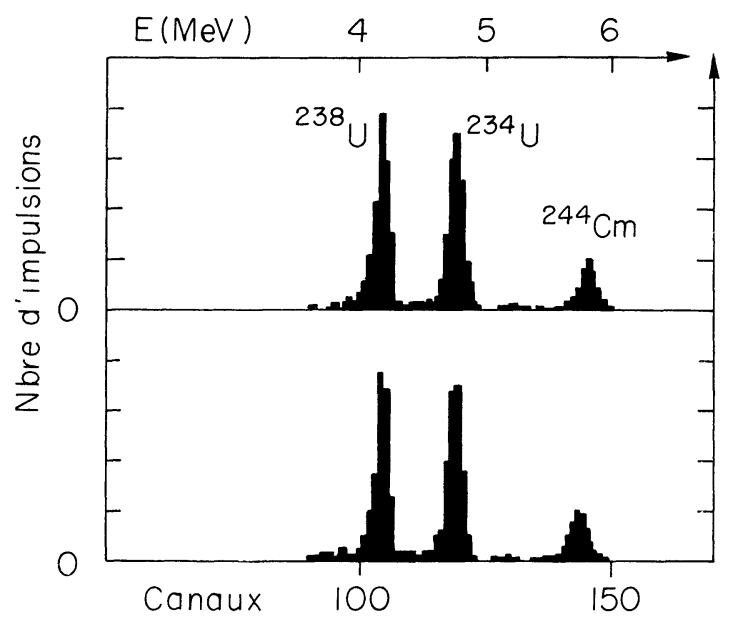

FIG. 2. - Spectre obtenu dans chaque chambre pour une source contenant les isotopes suivants : ${ }^{238 \mathrm{U}}(4,20$ et $4,15 \mathrm{MeV})$, ${ }^{234} \mathrm{U}(4,77$ et $4,72 \mathrm{MeV})$ et ${ }^{244} \mathrm{Cm}(5,81$ et $5,77 \mathrm{MeV})$. male trouvée sur la raie à $6,05 \mathrm{MeV}$ de ${ }^{212} \mathrm{Bi}$ est égale à $100 \mathrm{keV}$. Pour la raie à $8,78 \mathrm{MeV}$ de ${ }^{212} \mathrm{Po}$, elle vaut $180 \mathrm{keV}$.

Le mouvement propre de chaque chambre, intégré entre 2 et $6 \mathrm{MeV}$ est égal à 0,4 impulsion $/ \mathrm{mn}$. Si on ne considère que la largeur en énergie sur laquelle s'étend un pic, ce bruit de fond est ramené à 0,03 impulsion/mn, pour les raies alpha d'énergie inférieure à $6 \mathrm{MeV}$. Au-dessus de $6 \mathrm{MeV}$, ce mouvement propre est dix fois plus faible.

La figure 2 montre le spectre alpha d'une même source composite mesurée dans chacune des deux chambres d'ionisation.

Remerciements. - Nous remercions J. Camon, M. F. Kugler et le Bureau d'Etudes, ainsi que l'Atelier de Mécanique de l'Institut de Physique Nucléaire, sans qui cet appareil n'aurait pu être réalisé.

\section{Bibliographie}

[1] Price W. J., Nuclear Radiation Detection, McGraw Hill (1958).

[2] Ghiorso A., JAfFey A. M., Robinson H. P. et WeissBOURD B. B. dans « The Transuranium Elements », édité par G. T. Seaborg, J. J. Katz et W. N. Manning, McGraw Hill (1949), p. 1226. 